\title{
EXTENDING CONNECTED OPERATORS TO COLOUR IMAGES
}

\author{
Adrian N. Evans and David Gimenez. \\ Department of Electronic \& Electrical Engineering \\ University of Bath, UK
}

\begin{abstract}
Connected operators are an important tool for the analysis of greyscale images. In extending them to colour and other vector images there are a number of issues that must be addressed, including the definition of extrema, region merging criterion and the preservation of idempotence. This paper reviews the recently proposed approaches to these problems and considers some of the choices which must be made in the design of effective colour connective operators. An evaluation of the noise reduction performance resulting from these choices is presented. The use of the colour connected sieves in conjunction with the watershed transform for image segmentation is also investigated.
\end{abstract}

Index Terms - Morphological operations, connected operators, colour scale-space sieves, image processing

\section{INTRODUCTION}

Mathematical morphology was originally developed by Matheron and Serra and its techniques provide an important set of tools for image analysis. The two fundamental morphological operations of erosion and dilation can be combined to form openings and closings, which have the important property of idempotency. The traditional approach to implementing openings (resp. closings) is to apply successive erosions and dilations (resp. dilations and erosions) using fixed structuring elements. This approach is advantageous when some a priori knowledge of the shape, size and orientation of image features is available.

Alternatively, when other feature attributes such as area, volume or moment of inertia are more appropriate, openings and closings can be implemented directly using connected operators. Connected openings and closings were first proposed in 1992 by Cheng and Venetsanopoulos [1] and subsequently as connected or area operators [2]. Compared with approaches employing a fixed structuring element, connected operators can select the structuring element of a given area that, according to some attribute, best matches the image structure at each pixel position. Efficient algorithms such as the pixel queue, max-tree [3] and union find [4] have made the use of these operators a far more attractive proposition. Connected operators were originally developed using area as the attribute to control the filtering. The use of other attributes was proposed in [5] and, more recently, the development of higher-dimensional attribute spaces reported [6].

The extension of mathematical morphological techniques to colour and other multichannel images has received much recent attention. In their review paper, Aptoula and Lefèvre provide an up-to-date overview and classification of multivariate morphology techniques [7]. To date, the vast majority of these methods have been developed for fixed structuring elements. Indeed, only three of the 98 references cited in [7] specifically address the problems of extending connected operators to multi-channel images.

The simplest way to apply morphological operations to multichannel images is marginal processing, in which each channel is processed independently. This has the advantage of allowing all the methods developed for greyscale morphology to be directly applied to any multivariate image. When the image to be processing is highly correlated, such as an RGB image, it is often necessary to first apply a change of colour space or other decorrelating transform, as marginal processing completely disregards any inter-channel correlation. Marginal processing can also alter the spectral composition of an image, creating new colours and edge jitter. A connected filter for colour images based on applying marginal ordering in the hue, saturation and value (HSV) colour space was proposed by Weber and Acton [8]. This approach attempts to overcome the absence of ordering in the hue channel by applying a rotational shift to the hue, such that the creation of new colours is minimised. The filter was shown to outperform RGB marginal connected filtering for impulsive noise reduction but is not vector preserving.

Marginal ordering is just one of a number of vector ordering schemes that can be applied in multivariate morphology. To avoid the creation of new vectors and to take account of inter-channel correlation, vector ordering schemes that consider all channels simultaneously can be employed. A more detailed discussion on vector ordering and its application in multivariate morphology can be found in [7] and the references therein. To date, there have only been a few attempts to extend connected filters to colour images using vector processing. Brunner and Soille propose an approach based on the application of seeded region growing to the quasi-flat zones (both extrema and non-extrema) of multichannel images [9]. Other approaches, that only process the extrema, include the convex colour sieve (CCS) [10] and the vector area morphology sieve (VAMS) [11]. More recently, an algorithmic evaluation of the CCS and the VAMS has been performed and additional colour connected sieve structures proposed $[12,13]$.

The format of the remainder of this paper is as follows. Section 2 discusses the algorithm of recently proposed colour connected sieves and details some of the design choices at each algorithmic step. In section 3 the effect that some of these choices have on the noise reduction performance is analysed. The use of the colour connected sieves as a pre-filtering step for watershed transform segmentation is also investigated. Finally, some conclusions are drawn in section 4.

\section{COLOUR CONNECTED SIEVES}

\subsection{Introduction}

Greyscale connected filters process an image by removing the connected components that are lighter or darker than their neighbours, providing they meet some attribute criterion. In practice, this corre- 
sponds to identifying and then merging extrema regions in the image. In greyscale images two types of extrema are present and to produce a scale-space representation the maxima and minima must be combined, typically using an alternating sequential filter structure. Alternatively, the maxima and minima can be processed separately and then combined once the desired scale or attribute limit has been reached.

The CCS and the VAMS both process extrema without attempting to further classify them as maxima or minima. This approach is advantageous as is simplifies the processing and also reflects the fact that the identification of extrema in multivariate data is more straightforward than distinguishing between maxima and minima. For example, in Fig. 1 the vector $(8,2)$ is the natural outlier but is difficult to classify as either the maximum or the minimum.

Algorithmically, the CCS and VAMS colour connected sieves follow the same steps, as detailed below:

1. Identify all extremal regions;

2. Merge scale 1 extrema with the neighbouring region with the closest colour;

3. Repeat the previous 2 steps until no scale 1 extrema remain;

4. Repeat the previous 3 steps with increasing scale.

Steps 1, 2 and 4 of the above algorithm correspond to a greyscale connected sieve. Colour connected sieves additionally require step 3 , to take account of the fact that the merging process may create new extrema in multi-channel images. Step 3 therefore ensures that any new extrema with area less than the current scale do not survive, thus ensuring idempotence. In the above colour connected sieve algorithm, the key choices that must be made are (i) how to identify extrema in step 1; (ii) how to selected the region with closest colour in step 2 and (iii) the colour to assign to the merged regions. These three choices are discussed in more detail below.

\subsection{Extrema definition and merging rules}

As a consequence of the repetition of algorithm steps $1-2$ at each scale, the overall sieve performance greatly depends on how the colour extrema are identified. Unlike the greyscale case, where the definition of extrema is unambiguous, in multivariate images different extrema definitions give rise to different proportions of regions being classified as extremal, which in turn controls the aggressiveness of the filter. The CCS constructs a convex hull of each region and its connected neighbours and then defines a region as extremal if it lies on the edge of the hull [10]. This definition results in a very aggressive filter, as it classifies the vast majority of regions as extremal [13]. Taking Fig. 1 as an example, it can be seen that all 5 vectors are extrema.

An alternative vector extrema definition uses aggregate or reduced ordering. For a set of $n$ vectors $\mathcal{N}$, the vector extremum $\vec{x}_{V E}$ is defined such that

$$
\sum_{\vec{x}_{i} \in \mathcal{N}}\left\|\vec{x}_{V E}-\vec{x}_{i}\right\|_{p} \geq \sum_{\vec{x}_{i} \in \mathcal{N}}\left\|\vec{x}_{j}-\vec{x}_{i}\right\|_{p} \quad \forall j \in \mathcal{N}
$$

This definition has also been termed the vector outlier [14] and the most spectrally singular pixel [15]. The aggregate distances, given in the caption of Fig. 1, clearly identify the vector $(8,2)$ as the extremum.

Although (1) can find the vector extremum of a given vector set, there are two problems with directly using it to identify extrema in multichannel images. Firstly, the definition is non-unique. Secondly, image extrema correspond to flat zones whose measure of extremeness, for example intensity in greyscale image, is greater (or less)

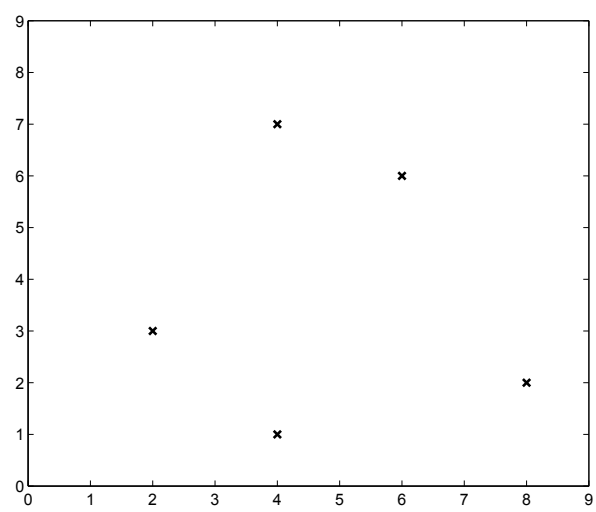

Fig. 1. Example vector set $(2,3),(4,1),(4,7),(6,6)$ and $(8,2)$ with aggregate differences of 18.38, 18.34, 19.11, 17.09 and 21.08.

than those of their neighbours and the set of vectors formed by each flat zone and its connected neighbours is different for neighbouring flat zones. The VAMS overcomes these problem by first using the right hand side of (1) to calculate the aggregate distance between each flat zone and its connected neighbours. The aggregate distances for each region form a scalar image whose regional maxima can be used to identify the vector extrema. As each region's aggregate distance depends on the number of its connected neighbours, the VAMS includes the additional step of scaling the aggregate distance of each flat zone by its area before determining the extrema. An alternative normalisation that uses perimeter instead of area has also been proposed [13].

Whereas the maxima in the aggregate distance image correspond to greyscale maxima and minima, the minima are those flat zones that are closer to their connected neighbours than any of their neighbouring regions. Applying connected closings will merge regions with colours similar to their neighbours. Employing both openings and closings increases the number of extrema, that act as seeds for the region merging process, and hence the aggressiveness of the sieve. This is achieved by the vector area morphology open-close sieve (VAMOCS) $[13,16]$. The multichannel connected closings are similar in spirit to the quasi-flat zones of [9] and their incorporation in the VAMOCS results in scale-space trees that show a closer correspondence with image objects and are more robust to image noise than those produced by the VAMS $[12,13]$. As the VAMOCS openings and closings operate on different image regions, there are only minor differences between open-close and close-open filters, particularly at lower scales.

There are many alternative distance metrics that can be used both instead of an $L_{p}$ norm in (1) and to determine the closest colour in step 2 of the colour connected sieve algorithm. Once such metric is the angular difference between two vectors given by $L_{\theta}=$ $\cos ^{-1}\left[\left(\vec{x}_{i} \cdot \vec{x}_{j}\right) /\left(\left\|\vec{x}_{i}\right\|_{p}\left\|\vec{x}_{j}\right\|_{p}\right)\right]$. Combined metrics consider both magnitude and direction, thus capturing the advantages of both properties. For example, [17] normalises the direction and magnitude differences by their maximum possible values to give the combined metric

$$
L_{a e 1}=1-\left[1-\frac{2}{\pi} L_{\theta}\right]\left[1-\frac{\left\|\vec{x}_{i}-\vec{x}_{j}\right\|_{p}}{\sqrt{3 \cdot 255^{2}}}\right]
$$

When the intensity is very low, hue dominates the metric but is also unreliable. An alternative combined metric, denoted $L_{a e 2}$, takes the 
NMSE/1e-2

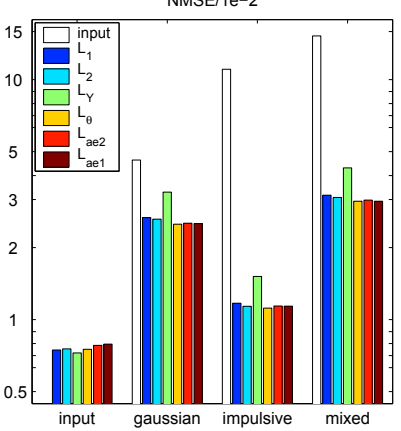

MCRE/1e-2

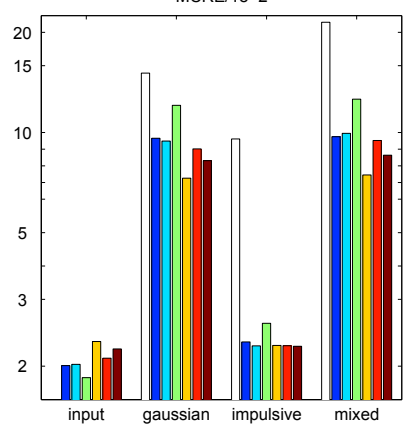

Fig. 2. VAMS results using various distance metrics to calculate the aggregate distances used for extrema calculation.

angle from $\mathrm{RGB}=$ [255 255255 ], if this is smaller than $L_{\theta}$ [12].

The final choice to be considered is the colour to assign to the merged regions. While greyscale connected operators simply take the colour of the non-extreme region, with colour images there are more choices. One option is to assign the colour of the largest of the two regions being merged [18]. Alternatively, the mean of the two regions can be used. Although this approach will not preserve the original vectors, it can be advantageous for noise reduction [7].

\section{EXPERIMENTAL RESULTS}

The first experiment evaluates the influence of the distance metric used to calculate the aggregate distance for extrema identification on the noise reduction performance. To this end, the $L_{1}$ and $L_{2}$ norms, the angular difference $\left(L_{\theta}\right)$ and the two combined metrics ( $L_{a e 1}$ and $L_{a e 2}$ ) were implemented in the VAMS. For comparison, the luminance difference $\left(L_{Y}\right)$ was also included. Regardless of the metric used for the aggregate distance, the region to merge with was selected using the $L_{2}$ norm. A set of six test images were corrupted by uncorrelated Gaussian $\left(\sigma^{2}=1000\right), 10 \%$ impulsive and mixed noise. The images were then sieved up to an area of 12 and the minimum normalised mean square error (NMSE) found. The average minimum NMSE and the corresponding mean chromaticity error (MCRE) given in Fig. 2 show all sieves to reduce noise to some degree, particularly impulsive noise. The luminance difference performs worst for all noise types, for both NMSE and MCRE. Both combined distance metrics perform well while the angular difference produces the lowest MCRE for Gaussian and mixed noise.

The influence of the distance metric used in the merging step on the noise reduction performance was evaluated using the set of six metrics from the previous experiment to select the region with the closest colour for the merging process, while fixing the $L_{2}$ norm for the aggregate differences. Cases where two or more colours were equidistant were resolved using the luminance. These results are presented in Fig. 3 and, in contrast to those of Fig. 2, show that the angular difference performs poorly, often worse than the luminance difference. Here, the $L_{1}$ norm performs best on impulsive noise and the $L_{2}$ norm has a slight advantage with Gaussian and mixed noise. Again, the relative noise reduction is greatest for impulsive noise.

Finally, the choice of colour to assign to the merged regions is investigated. In Fig. 4 the NMSE and MCRE for the standard VAMS, in which the merged region is assigned the colour of the nonextreme region, are compared to other choices, such as the mean and (marginal) median of the original colours in regions to be merged.
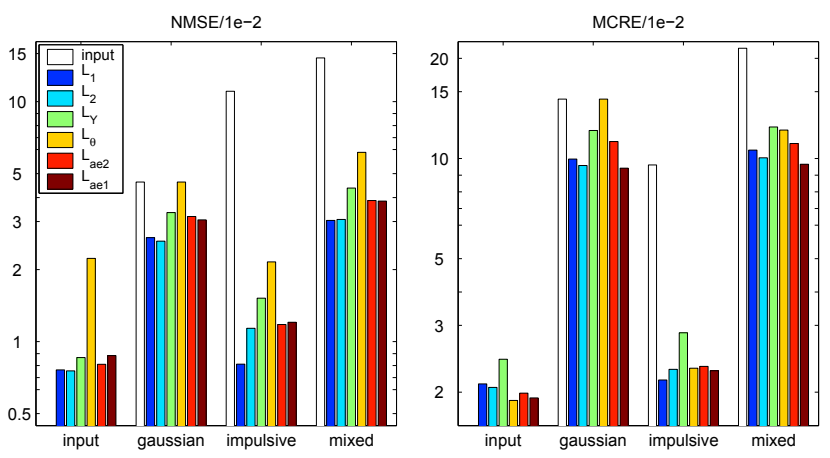

Fig. 3. VAMS results using various distance metrics to select the region for the extrema to merge with.

As neither of these options is vector preserving, a guided approach is also evaluated. In the guided approach a central vector (mean or median) is used as a "guide" and the closest input vector to the guide selected. Using the mean as the guide vector produces a lower NMSE than the median for Gaussian noise but is worse for impulsive and mixed noise. However, the standard VAMS outperforms both these and the non-guided techniques for all noise types. In other experiments, we have found this is the case for most colour connected sieves, the one exception being a mean-based CCS with Gaussian noise.

As the colour connected sieves generate an image partition they can be directly used for image segmentation. In practice this requires a suitable scheme for selecting sets of regions that produce a good segmentation performance, for example by using a global attribute limit or other tree-pruning strategy. An alternative approach is to use the VAMS in conjunction with the watershed transform. Fig. 5(b) shows the result of applying the watershed to the VAMS gradient of the image in Fig. 5(a), using the $L_{2}$ norm to calculate the aggregate distances. This result shows that the VAMS aggregate distance provides a suitable gradient for the watershed but also suffers from the watershed's characteristic over-segmentation. Using the VAMS as a pre-filter reduces the over-segmentation while retaining significant feature boundaries. This is evident in Fig. 5(c) and (d), where the attributes limits were area $=100$ and contrast $=100$, respectively. Here, the contrast was given by the maximum distance ( $L_{2}$ norm) between the original colours within the region and those of its neighbours. As the over-segmentation results from spurious minima in the gradient image, only the minima in the aggregate distance were sieved.
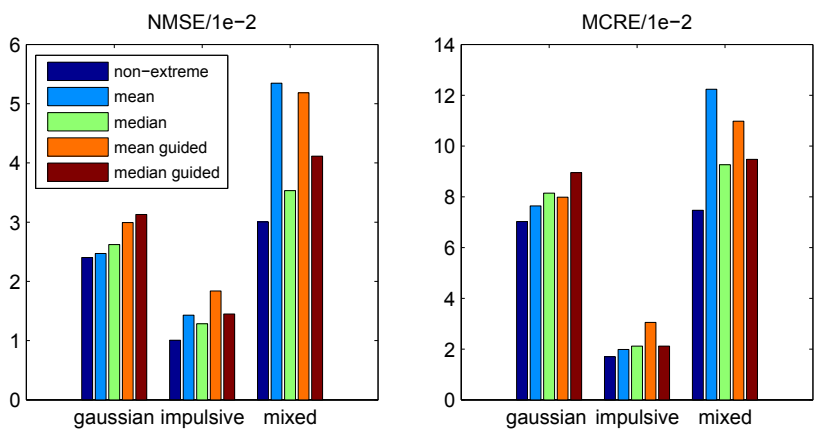

Fig. 4. VAMS results produced using different mechanisms to determine the colours of the merged regions. 


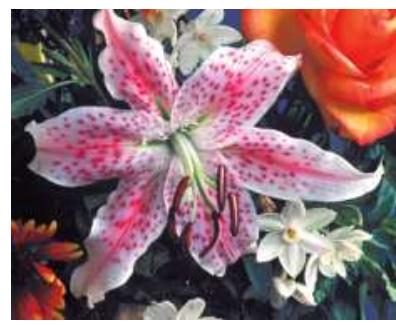

(a) Lily test image

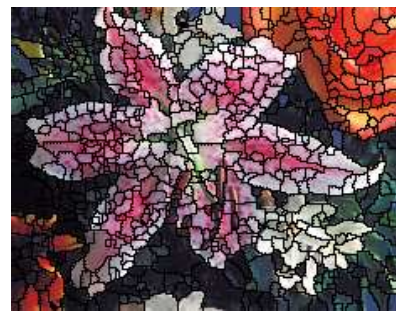

(c) Sieved to area $=100$

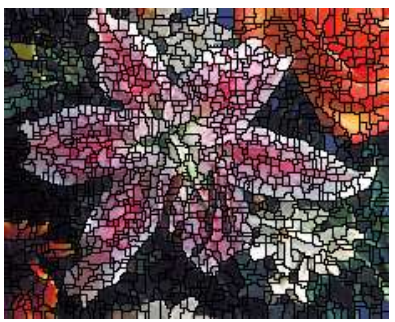

(b) No sieving

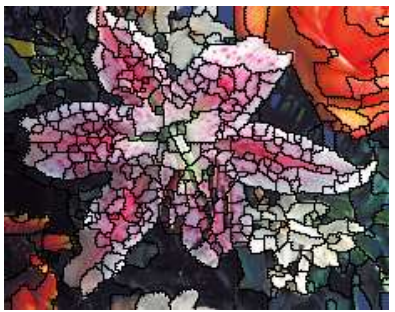

(d) Sieved to contrast $=100$
Fig. 5. Combined VAMS/watershed transform results using aggregate distance gradient.

\section{CONCLUSIONS AND DISCUSSION}

An analysis of recent extensions of connected operators to colour images has been presented and some of the design choices at the various algorithm steps detailed. For VAMS-type colour sieves these include the distance metric used for the extrema definition and merging process, and choice of colour to assign merged regions. An evaluation in terms of noise reduction shows the angular and combined distances to perform well for extrema definition, as do the $L_{1}$ and $L_{2}$ norms for selecting the region to merge with. The conventional approach to assigning the non-extreme region's colour to the merged region is the best choice for the vast majority of cases. The potential benefit of using colour connected sieving as a pre-processing stage for the watershed transform has also been demonstrated.

Pattern spectra are an important tool for image analysis and classification. A greyscale size pattern spectrum can be obtained by filtering with increasing scale and, at each scale, subtracting the sum of grey levels from the sum at the previous scale. Typically two spectra are calculated containing information about the light and dark image patterns, an approach not possible with colour connected sieves such as the VAMS that do not distinguish between extrema types. A possible way to overcome this problem is to generate a pseudo pattern spectrum by summing the absolute differences between the intensities at the current and previous scale. However, in contrast to the greyscale case, as new extrema can be created at each scale the sum at each step is not completely resolved until the scale reaches the total number of image pixels.

Our colour connected sieves implementations are based on the pixel queue algorithm and therefore their processing times inherit its dependency on image size and scale. Previously, we have shown that the additionally complexity associated with the identification and merging of new extrema at each scale results in an approximate doubling of the processing time, compared with marginal processing using the same algorithm [16]. However, if marginal processing is performed using faster algorithms, such as the union find, its computation advantage is much greater, particularly at higher scales.

\section{REFERENCES}

[1] F. Cheng and A.N. Venetsanopoulos, "An adaptive morphological filter for image processing," IEEE Trans. Image Processing, vol. 1, pp. 533-539, 1992.

[2] P. Salembier and J. Serra, "Flat zones filtering, connected operators, and filters by reconstruction," IEEE Trans. Image Processing, vol. 4, no. 8, pp. 1153-1160, August 1995.

[3] P. Salembier, A. Oliveras, and L. Garrido, "Antiextensive connected operators for image and sequence processing," IEEE Trans. Image Processing, vol. 7, no. 4, pp. 555-570, April 1998.

[4] A. Meijster and M.H.F. Wilkinson, "A comparison of algorithms for connected set openings and closings," IEEE Trans. Pattern Analysis and Machine Intelligence, vol. 24, no. 4, pp. 484-494, April 2002.

[5] E.J. Breen and R. Jones, "Attribute openings, thinnings, and granulometries," Computer Vision and Image Understanding, vol. 64, pp. 377-389, 1996.

[6] E.R. Urbach, N.J. Boersma, and M.H.F. Wilkinson, "Vectorattribute filters," in Proc. International Symposium on Mathematical Morphology, pp. 95-104. April, 2005.

[7] E. Aptoula and S. Lefèvre, "A comparative study on multivariate mathematical morphology," Pattern Recognition, vol. 40, no. 11, pp. 2914-2929, November 2007.

[8] K.R. Weber and S.T. Acton, "On connected filters in color image processing," Journal of Electronic Imaging, vol. 13, no. 3, pp. 619-629, 2004.

[9] D. Brunner and P. Soille, "Iterative area filtering of multichannel images," Image and Vision Computing, vol. 25, no. 8, pp. 1352-1364, Aug. 2007.

[10] S. Gibson, R. Harvey, and G.D. Finlayson, "Convex colour sieves," in Proc. 4th Int. Conf. on Scale Space Methods in Computer Vision, vol. LNCS 2695, pp. 550-563, 2003.

[11] A.N. Evans, "Vector area morphology for motion field smoothing and interpretation," IEE Proceedings Vision, Image and Signal Processing, vol. 150, no. 4, pp. 219-226, August 2003.

[12] D. Gimenez, Colour morphological sieves for scale-space image processing, Ph.D. thesis, University of Bath, 2007.

[13] D. Gimenez and A.N. Evans, "An evaluation of area morphology scale-spaces for colour images," Computer Vision and Image Understanding, vol. 110, pp. 32-42, April 2008.

[14] S.-Y. Zhu, K.N. Plataniotis, and A.N. Venetsanopoulos, "Comprehensive analysis of edge detection in color image processing," Optical Engineering, vol. 38, no. 4, pp. 612-625, April 1999.

[15] A. Plaza, P. Martínez, R. Pérez, and J. Plaza, "Spatial/spectral endmember extraction by multidimensional morphological operations," IEEE Trans. Geosciences and Remote Sensing, vol. 40, no. 9, pp. 2025-2041, 2002.

[16] D. Gimenez and A.N. Evans, "Colour morphological scalespaces for image segmentation," in Proc. British Machine Vision Conference, 2005, pp. 909-918.

[17] D. Androutsos, K.N. Plataniotis, and A.N. Venetsanopoulos, "Distance measures for color image retrieval," in IEEE Int. Conf. on Image Processing, 1998, pp. Vol II: 770-774.

[18] P. Salembier and L. Garrido, "Binary partition tree as an efficient representation for image processing, segmentation, and information retrieval," IEEE Trans. Image Processing, vol. 9, no. 4, pp. 561-576, April 2000. 